\title{
Prognostic Value of Circulating Tumor Cells in Ovarian Cancer: A Meta-Analysis
}

\author{
Yunlan Zhou ${ }^{\circledR}$, Bingxian Bian ${ }^{\circledR}$, Xiangliang Yuan, Guohua Xie, Yanhui Ma, Lisong Shen* \\ Department of Clinical Laboratory, Xin Hua Hospital, Shanghai Jiao Tong University School of Medicine, \\ Shanghai 200092, China \\ ه These authors contributed equally to this work. \\ * lisongshen@ hotmail.com
}

\section{Abstract}

\section{Background}

The prognostic value of circulating tumor cells (CTCs) in ovarian cancer has been investigated in previous studies, but the results are controversial. Therefore we performed a metaanalysis to systematically review these data and evaluate the value of CTCs in ovarian cancer.

\section{open access}

Citation: Zhou Y, Bian B, Yuan X, Xie G, Ma Y, Shen $L$ (2015) Prognostic Value of Circulating Tumor Cells in Ovarian Cancer: A Meta-Analysis. PLOS ONE 10 (6): e0130873. doi:10.1371/journal.pone.0130873

Editor: Jeffrey Chalmers, The Ohio State University, UNITED STATES

Received: December 16, 2014

Accepted: May 26, 2015

Published: June 22, 2015

Copyright: $\odot 2015$ Zhou et al. This is an open access article distributed under the terms of the Creative Commons Attribution License, which permits unrestricted use, distribution, and reproduction in any medium, provided the original author and source are credited.

Data Availability Statement: All relevant data are within the paper and its Supporting Information files

Funding: This work was supported by National Natural Science Foundation of China (Lisong Shen 81372641, Yunlan Zhou 81401946, Xiangliang Yuan 81472244)

Competing Interests: The authors have declared that no competing interests exist.

\section{Materials and Methods}

A literary search for relevant studies was performed on Embase, Medline and Web of Science databases. Then pooled hazard ratios (HRs) for survival with $95 \%$ confidence intervals (Cls), subgroup analyses, sensitivity analyses, meta-regression analyses and publication bias were conducted.

\section{Results}

This meta-analysis is based on 11 publications and comprises a total of 1129 patients. The prognostic value of the CTC status was significant in overall survival (OS) (HR, 1.61;95\% $\mathrm{Cl}, 1.22-2.13$ ) and progression-free survival (PFS)/disease-free survival (DFS) (HR, 1.44; $95 \% \mathrm{Cl}, 1.18-1.75)$. Furthermore, subgroup analysis revealed that the value of CTC status in OS was significant in "RT-PCR" subgroup (HR, 2.02; 95\% Cl, 1.34-3.03), whereas it was not significant in "CellSearch" subgroup (HR, 1.15; 95\% Cl 0.45-2.92) and "other ICC" subgroup (HR, 1.09; 95\% $\mathrm{Cl} 0.62-1.90)$. The presence of CTC was also associated with an increased CA-125 (OR, 4.07; 95\% Cl, 1.87-8.85).

\section{Conclusion}

Our study demonstrates that CTC status is associated with OS and PFS/DFS in ovarian cancer. 


\section{Introduction}

Ovarian cancer is the second leading cause of death among gynecologic malignancies in the world [1] owing to the fact that a majority of patients are diagnosed in late stages of the disease [2]. In such a setting, identifying prognostic indicators for patients with ovarian cancer is crucial. CA-125 is a frequently used biomarker in ovarian cancer, but some non-malignant conditions also cause elevated serum CA-125 concentrations [3]. Thus additional prognostic markers are urgently needed for ovarian cancer.

CTCs are tumor cells that have shed into bloodstream from the primary tumors, recurrences, or metastases, and possess antigenic and genetic tumor-specific characteristics. Two major detection methods have been used to identify CTC, including immunocytochemistry (ICC), reverse-transcriptase polymerase chain reaction (RT-PCR). US Food and Drug Administration (FDA) only approved the CellSearch system for clinical use currently, which enriches and detects CTC of epithelial origin by means of ICC methods. There is no published polymorphism studies associated with CTCs in ovarian cancer in previous studies.

CTCs have been demonstrated to have prognostic value among patients with breast, colorectal, gastric, lung and pancreatic cancers in previous meta-analyses [4-8]. However, the value of CTCs in ovarian cancer still remains controversial. Some studies did not observe any correlation between CTC status and prognosis. In contrast, other studies revealed association between CTC status and prognosis. After considering the conflicting results from previous studies, we performed the first meta-analysis to investigate the prognostic value of CTCs on OS and DFS/PFS in patients with ovarian cancer confirmed by histopathologic examinations. Furthermore, subgroup analyses were conducted to evaluate whether the detection method and treatment methods influence the prognostic value of CTCs.

\section{Methods}

\section{Search strategy}

A literary search for potential studies was performed on Embase (from 1974 to November 1, 2014), Medline (from 1966 to November 1, 2014) and Web of Science databases including Science Citation Index Expanded, Social Sciences Citation Index, Arts \& Humanities Citation Index, Conference Proceedings Citation Index-Science, Conference Proceedings Citation Index-Social Science \& Humanities, Current Chemical Reactions (from 1985 to November 1, 2014). Search term combinations were"ovarian cancer,"'ovary cancer,"'"ovarian carcinoma,"'ovary carcinoma, "' ovarium carcinoma,"'ccirculating tumor cells,"'circulating tumor cell,"'circulating cancer cells,"“circulating cancer cell,"“CTCs" and"CTC" in title/abstract. Relevant articles were also screened manually to prevent omission of any research. If the data in studies were insufficient, we contacted authors by e-mail. And we subsequently excluded the studies when authors couldn't be contacted.

\section{Selection criteria and quality assessment}

Studies were selected from initial search using following inclusion criteria: (1) survival data were analyzed for the prognostic value of CTCs in ovarian cancer; (2) sufficient data were provided to determine $\mathrm{HR}$ with $95 \%$ confidence interval (CI); (3) when the same study population was published at several reports, only the most complete one was selected for our meta-analysis; (4) more than 30 patients were enrolled in each study; (5) reports in English were eligible. Studies with reviews, letters, editorials, abstracts, comments and case reports were also excluded. 
Two investigators independently evaluated the quality of included studies using the Newcastle-Ottawa Scale (NOS). A score of zero points meant the study had the worst quality, whereas a score of nine points meant the study had the best quality. When disagreements occured, they were solved by discussion. We also conducted subgroup and sensitivity analyses to assess study quality.

\section{Data extraction}

Following details were extracted from included study: name of first author, year of publication, patients' country, number of patients, disease stage, CTC detection methods, target antigen/ target gene, cutoff defining positivity of CTC, HRs with 95\% CIs for PFS, DFS, OS. HRs and 95\% CIs were extracted from multivariable analyses. When they were not directly extracted from the original study, they were calculated by the method of Tierney et al.[9]. When more than 1 blood sample per patient was detected at different time points such as baseline, midtherapy and post-therapy, we only investigated baseline value of CTC in ovarian cancer.

\section{Statistical analysis}

As the detection methods of CTCs, and detection rates of CTC in patients were very different across studies, a random effect model was used for calculating the pooled HR [10,11]. In order to evaluate potential sources of heterogeneity, subgroup analyses were performed by detection methods and treatment methods. Stratified analysis with respect to population was not conducted because most of the included studies were from Caucasian countries. Meta-regression analyses were also used to evaluate potential causes of heterogeneity (a p-value $<0.05$ was considered statistically significant). To evaluate the influence of single studies on the pooled HRs, we performed a sensitivity analysis by estimating the average HR in the absence of each study. To investigate whether a publication bias might have affected the validity of the estimates [12], we performed Begg's funnel plot and Egger's linear regression test (a p-value $<0.05$ was considered statistically significant). STATA version 12.0 was performed to process all of the data.

\section{Results}

\section{Characteristics of identified studies}

The study followed the criterions for systematic review and meta-analysis of genetic association studies (S1 and S2 Checklists). The flowchart of search strategy for articles is presented in Fig 1 and S1 Table. 295 articles related to the keywords were initially reviewed. Of these articles, 280 were excluded after screening of titles, keywords and abstracts because they were obviously irrelevant studies, duplicates, reviews, abstracts and comments. Another 4 articles were excluded after reviewing the full texts because of insufficient data, multiple publications and small sample size, leaving 11 eligible studies [13-23]. published between 2002 and 2014. As shown in Table 1, a total of 1129 patients (ranging from 30 to 216 for individual study) were included in our meta-analysis. The detection rate of CTCs in these patients ranged from $12 \%$ to $83 \%$. Most of studies were from Caucasian countries [13-16,18,20-23]. One study was from Asian countries [17] and the other one was from multi-country[19]. Methods used to detect CTCs were CellSearch system, other ICC and the RT-PCR. 64 women with cancer were included in Judson's study [15], but only 51 patients with new diagnosis were included in the Kaplan-Meier distributions. 60 patients were enrolled in Behbakh's study, whereas only 43 patients were included in the Kaplan-Meier PFS distributions for pre-treatment CTCs [14]. HRs with $95 \%$ CIs were directly extracted from original articles in three studies $[17,19,23]$. HRs with $95 \%$ CIs were not directly reported in eight studies [13-16,18,20-22] and calculated from 


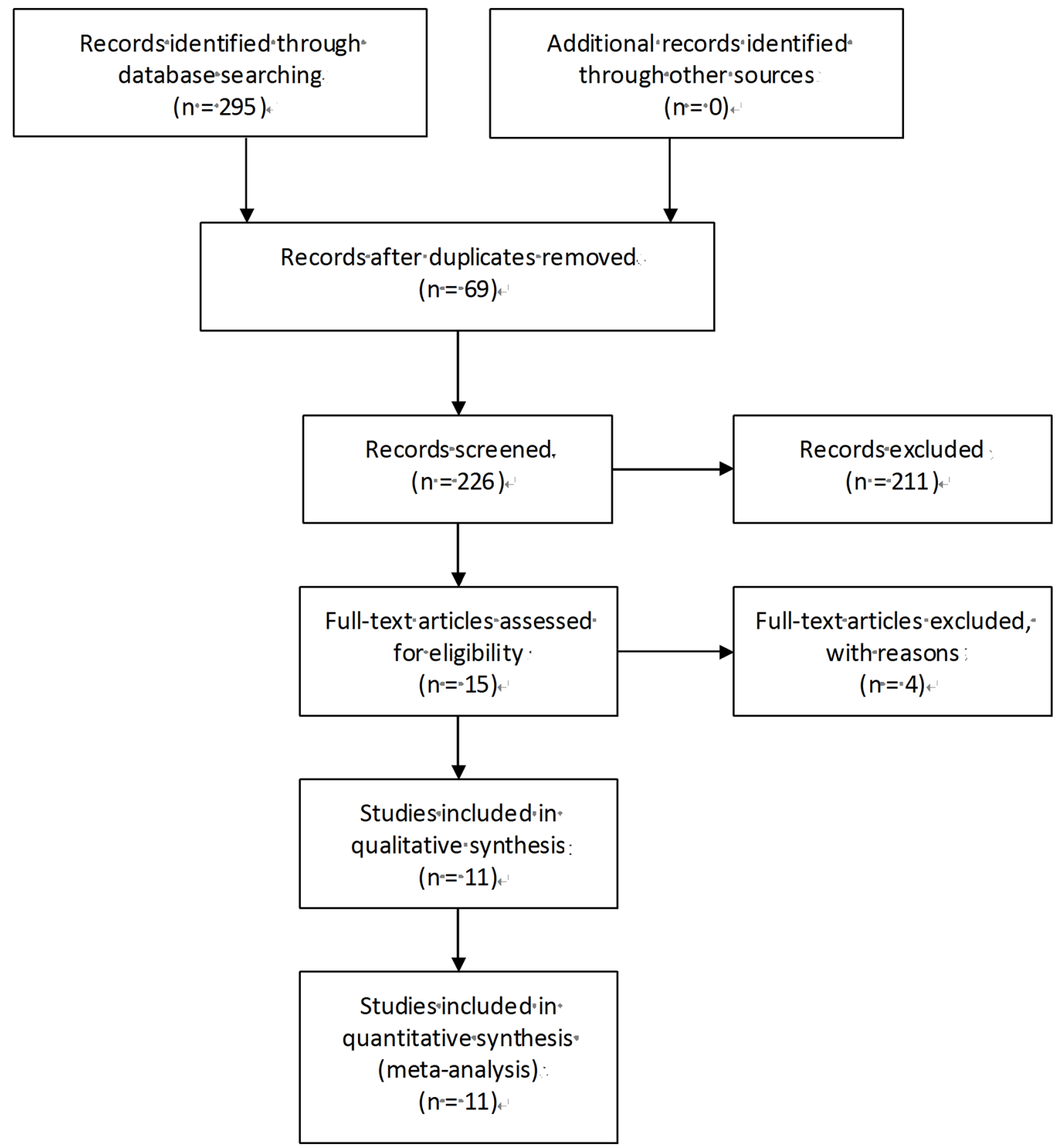

Fig 1. PRISMA flow Chart of literature search and study selection.

Kaplan-Meier curves suggested by Tierney et al.[9]. Quality assessment of included studies is shown in Table 2.

\section{CTC and OS}

Pooled HR. HRs for PFS/DFS were available in ten studies[13,15-23]. The pooled HR showed a significantly increased risk of mortality in patients with CTC positive group (HR, 1.61; 95\% CI,1.22-2.13; Fig 2). Heterogeneity among studies was not noted $\left(\mathrm{P}=0.207\right.$ and $\mathrm{I}^{2}=$ $25.7 \%)$. 
Table 1. Main characteristics of the eligible studies.

\begin{tabular}{|c|c|c|c|c|c|c|}
\hline Study & $\begin{array}{l}\text { No. of } \\
\text { patients }\end{array}$ & $\begin{array}{l}\text { Tumor } \\
\text { stage }^{\text {a }}\end{array}$ & $\begin{array}{l}\text { Detection } \\
\text { Method }\end{array}$ & Target antigen/target gene & $\begin{array}{l}\text { Detection rate, } \\
\%\end{array}$ & $\begin{array}{l}\text { Cutoff of } \\
\text { CTCs }\end{array}$ \\
\hline Marth 2002[13] (Norway) & 90 & I-IV (77.8) & Other ICC & Pancarcinoma/epithelial glycoprotein & $12 \%$ & - \\
\hline Judson 2003[15] (America) & 51 & I-IV (NR) & Other ICC & $\begin{array}{l}\text { Keratin } 8 \text { and 18,TFS-2,CK-7,CK-20, } \\
\text { EGFR }\end{array}$ & $21.6 \%$ & - \\
\hline Fan 2009[20] (America) & 66 & I-IV (78.8) & Other ICC & CAM,Epi & $34.8 \%$ & $1 \mathrm{CTC} / 3 \mathrm{~mL}$ \\
\hline $\begin{array}{l}\text { Poveda 2011[19] (Multi- } \\
\text { country) }\end{array}$ & 216 & NR (NR) & Cellsearch & СК,ЕрСАМ & $14.4 \%$ & $\begin{array}{l}2 \mathrm{CTC} / 7.5 \\
\mathrm{~mL}\end{array}$ \\
\hline $\begin{array}{l}\text { Behbakht 2011[14] } \\
\text { (America) }\end{array}$ & 43 & $\mathrm{NR}(\mathrm{NR})$ & Cellsearch & СК,ЕрСАМ & $44 \%$ & $\begin{array}{l}2 \mathrm{CTC} / 7.5 \\
\mathrm{~mL}\end{array}$ \\
\hline Aktas 2011[21] (Germany) & 122 & I-IV (NR) & RT-PCR & EpCAM, MUC-1, HER2, CA 125 & $19 \%$ & - \\
\hline $\begin{array}{l}\text { Obermayr 2013[22] } \\
\text { (Europe) }\end{array}$ & 200 & II-IV (96) & RT-PCR & PPIC & $17 \%$ & - \\
\hline Liu 2013[16] (America) & 30 & I-IV (100) & Cellsearch & СК,ЕрСАМ & $60 \%$ & $\begin{array}{l}2 \mathrm{CTC} / 7.5 \\
\mathrm{~mL}\end{array}$ \\
\hline Sang 2014[17] (China) & 80 & I-IV (88.8) & RT-PCR & MAGE-A & $47.5 \%$ & - \\
\hline Pearl 2014[18] (America) & 88 & I-IV (80.7) & Other ICC & Epi,HL & $83.0 \%$ & $5 \mathrm{CTC} / 1 \mathrm{~mL}$ \\
\hline $\begin{array}{l}\text { Kuhlmann 2014[23] } \\
\text { (Germany) }\end{array}$ & 143 & I-III (52) & RT-PCR & EpCAM, MUC1, MUC16 & $14 \%$ & - \\
\hline
\end{tabular}

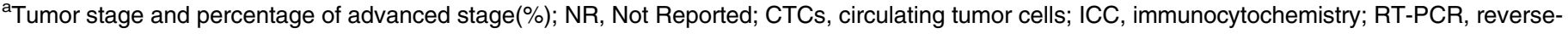
transcriptase polymerase chain reaction.

doi:10.1371/journal.pone.0130873.t001

Table 2. Main results.

\begin{tabular}{|c|c|c|c|c|}
\hline Author & Outcome & HR & $95 \% \mathrm{Cl}$ & NOS score \\
\hline \multirow[t]{2}{*}{ Marth 2002} & OS & 1.99 & $0.23-17.16$ & 6 \\
\hline & PFS & 2.19 & $0.84-5.74$ & \\
\hline \multirow[t]{2}{*}{ Judson 2003} & OS & 2.14 & $0.37-12.29$ & 7 \\
\hline & PFS & 1.45 & $0.55-3.83$ & \\
\hline \multirow[t]{2}{*}{ Fan 2009} & OS & 0.89 & $0.40-1.95$ & 7 \\
\hline & DFS & 1.44 & $0.78-2.64$ & \\
\hline \multirow[t]{2}{*}{ Poveda 2011} & OS & 1.54 & $0.93-2.54$ & 7 \\
\hline & PFS & 1.58 & $0.99-2.53$ & \\
\hline Behbakht 2011 & PFS & 1.61 & $0.79-3.29$ & 6 \\
\hline \multirow[t]{2}{*}{ Aktas 2011} & OS & 4.56 & $1.94-10.73$ & 6 \\
\hline & PFS & 1.58 & $0.86-2.88$ & \\
\hline \multirow[t]{2}{*}{ Obermayr 2013} & OS & 2.04 & $1.17-3.54$ & 7 \\
\hline & DFS & 1.35 & $0.90-2.03$ & \\
\hline \multirow[t]{2}{*}{ Liu 2013} & OS & 0.53 & $0.12-2.40$ & 7 \\
\hline & PFS & 0.71 & $0.30-1.69$ & \\
\hline Sang 2014 & OS & 1.40 & $0.87-2.27$ & 6 \\
\hline \multirow[t]{2}{*}{ Pearl 2014} & OS & 1.06 & $0.41-2.73$ & 6 \\
\hline & PFS & 1.21 & $0.49-2.97$ & \\
\hline \multirow[t]{2}{*}{ Kuhlmann 2014} & OS & 1.85 & $1.03-3.32$ & 7 \\
\hline & PFS & 1.5 & $0.81-2.79$ & \\
\hline
\end{tabular}

HR, hazard ratio; Cl, confidence intervals; NOS, Newcastle-Ottawa Scale; OS, overall survival; PFS,progression-free survival; DFS,disease-free survival. doi:10.1371/journal.pone.0130873.t002 
Study

ID

ES $(95 \% \mathrm{Cl})$

$\%$

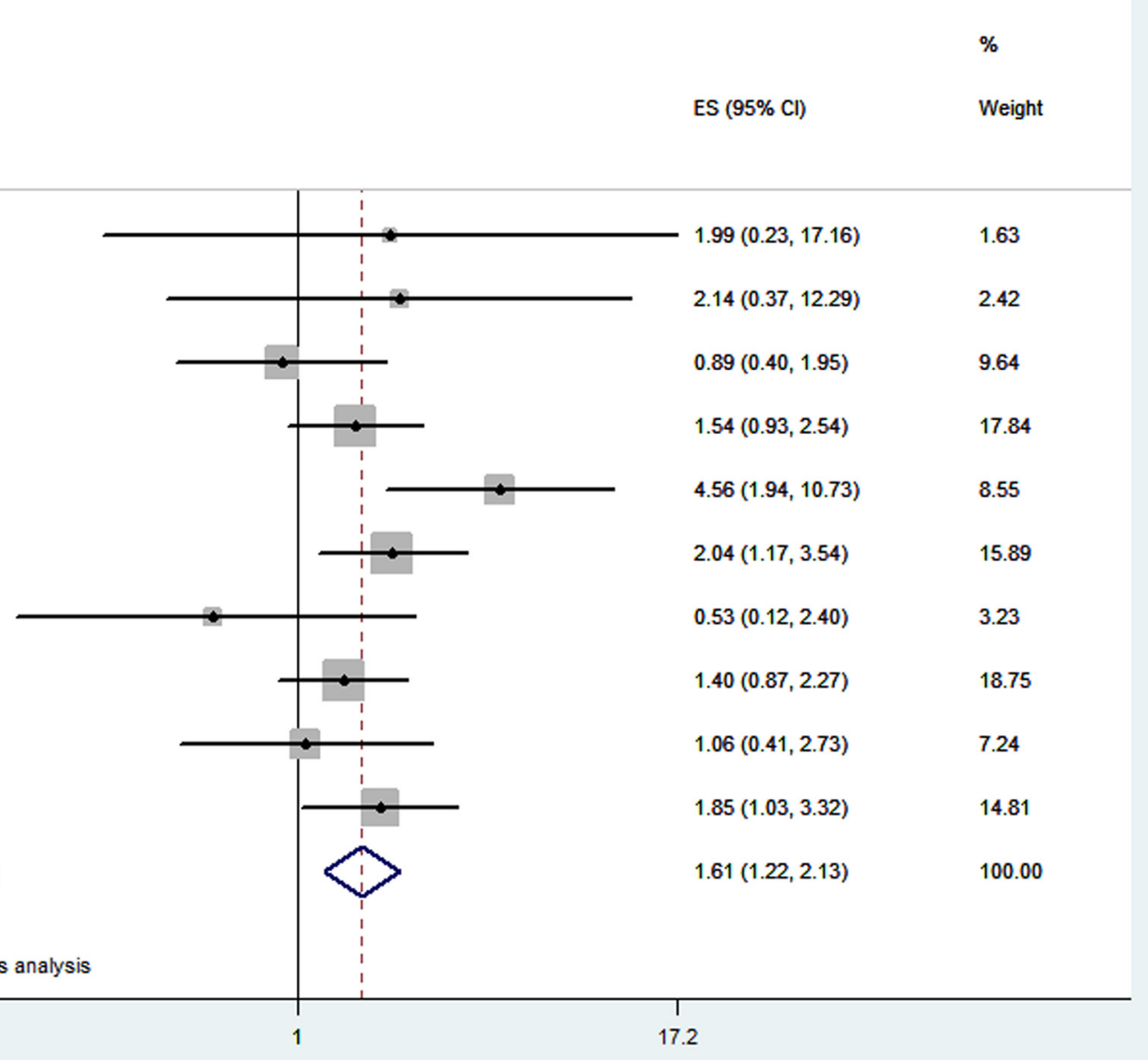

Fig 2. Forest plot showing the meta-analysis of hazard ratio estimates for OS in overall patients. OS = overall survival.

doi:10.1371/journal.pone.0130873.g002 
Study

ID
$\%$

ES $(95 \% \mathrm{Cl}) \quad$ Weight

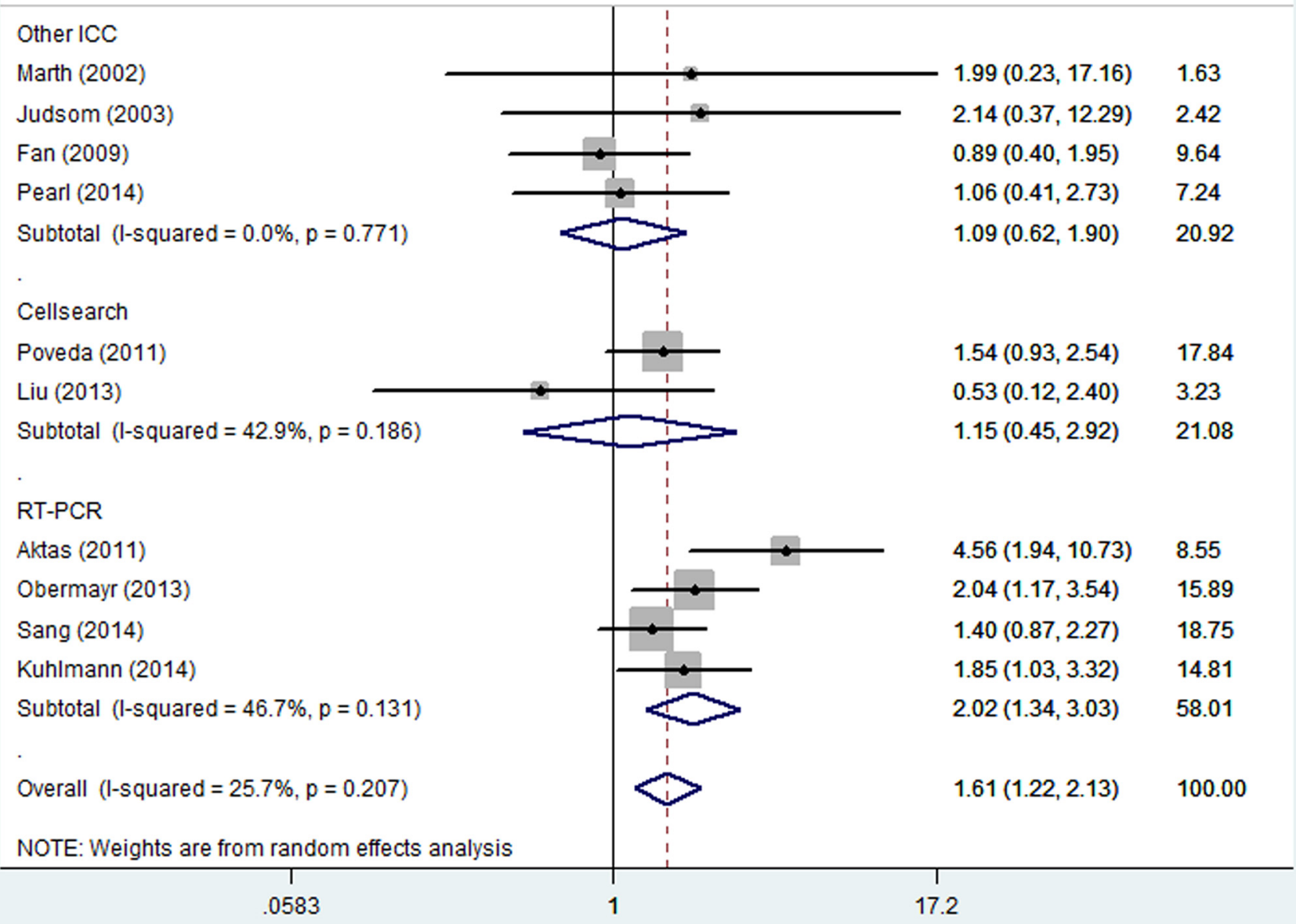

Fig 3. Forest plot showing the meta-analysis of hazard ratio estimates for OS in "Other ICC" subgroup, "Cellsearch" subgroup and "RT-PCR" subgroup. Subgroup analysis based on different CTC detection methods. OS = overall survival; ICC = immunocytochemistry; RT-PCR = reversetranscriptase polymerase chain reaction; CTC = circulating tumor cell.

doi:10.1371/journal.pone.0130873.g003

\section{CTC and PFS/DFS}

Pooled HR. HRs for PFS/DFS were available in ten studies [13-16,18-23]. The estimated pooled HR showed an increased risk of disease progression in patients with CTC positive group (HR, 1.44; 95\%CI, 1.18-1.75; Fig 5). The heterogeneity among studies was not noted $\left(\mathrm{P}=0.918, \mathrm{I}^{2}=0.0 \%\right)$.

\section{Meta-regression analyses}

We conducted the meta-regression analysis based on the following covariates: percentage of advanced stage (stage IV or stage III), detection method (ICC vs. RT-PCR), publication year, treatment type (Surgery vs. Chemotherapy) and sample size ( $>=90$ vs. $<90$ ). As shown in S2 
Study

ID
ES $(95 \% \mathrm{Cl})$

Weight

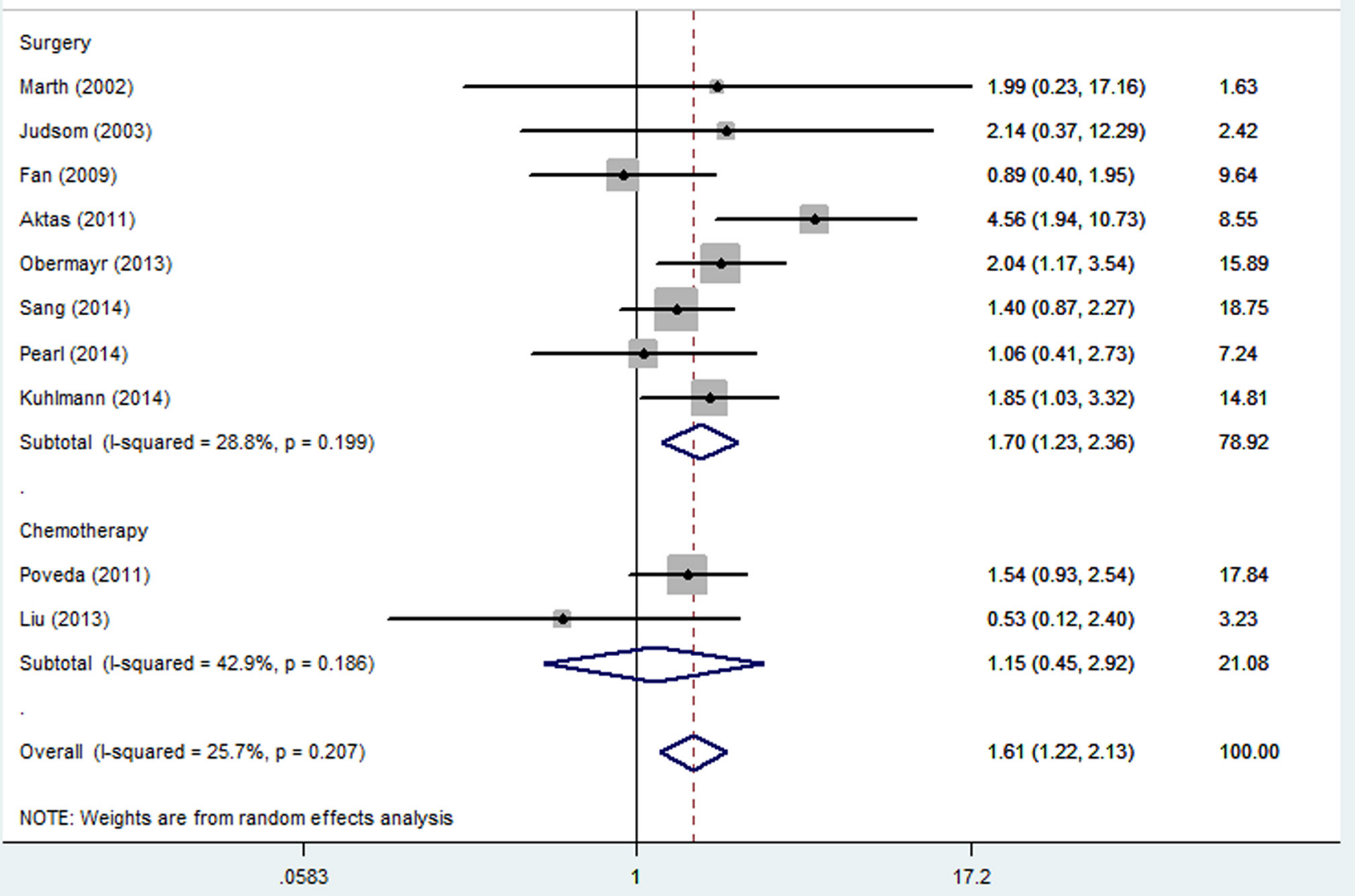

Fig 4. Forest plot showing the meta-analysis of hazard ratio estimates for OS in "Surgery" subgroup and "Chemotherapy" subgroup. Subgroup analysis based on different treatment methods. OS = overall survival.

doi:10.1371/journal.pone.0130873.g004

Table, no significant association could be observed in percentage of advanced stage $(\mathrm{p}=0.645)$, detection method (0.118), publication year (0.619), treatment type(0.469) and sample size (0.059).

\section{CTC and CA-125}

CA-125 is also a useful prognostic tumor marker for survival in ovarian cancer. Five studies reported data on correlation between the presence of CTC and elevated CA-125. Of these, one study [17] confirmed the correlation between CTC and CA-125. Conversely, four studies [1820,22] suggested no correlation between CTC and CA-125. Pooled outcome from the five studies demonstrated a strong correlation between presence of CTC and increased CA-125 (OR, 4.07; 95\%CI, 1.87-8.85; Fig 6). 
Study

ID
$\%$

ES $(95 \% \mathrm{Cl}) \quad$ Weight

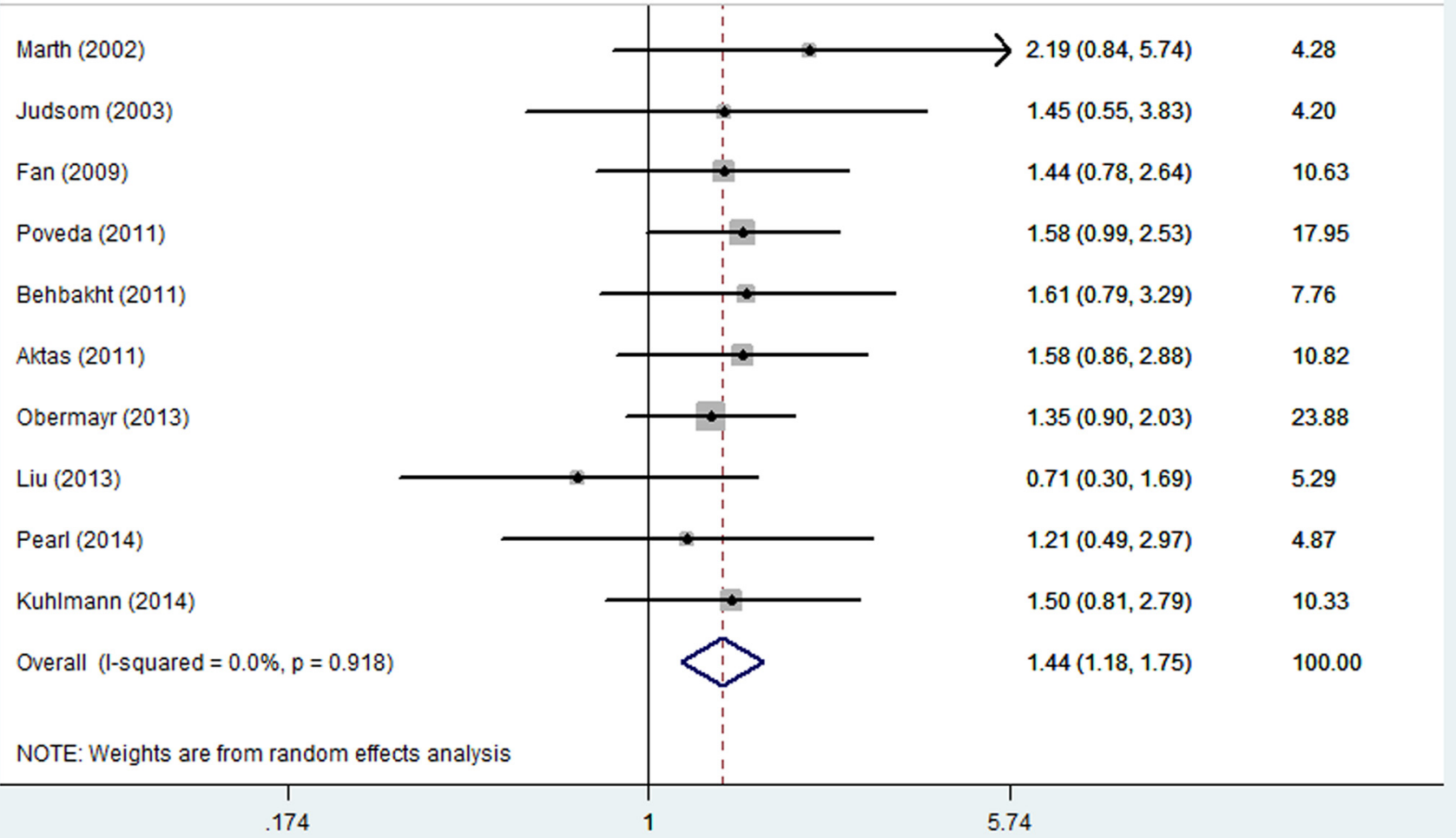

Fig 5. Forest plot showing the meta-analysis of hazard ratio estimates for DFS/PFS in overall patients. DFS = disease-free survival; PFS = progression-free survival.

doi:10.1371/journal.pone.0130873.g005

\section{Sensitivity analyses and publication bias}

In order to evaluate the influence of single studies on the pooled HRs, we performed a sensitivity analysis by estimating the average HR in the absence of each study. The results indicated that no individual studies significantly influenced the pooled HRs (Fig 7). Begg's funnel plot $(\mathrm{P}=1$, Fig 8$)$ and Egger's linear regression test $(\mathrm{P}=0.806)$ did not show evidence for publication bias.

\section{Discussion}

Many studies have reported the prognostic value of CTCs in patients with ovarian cancer, and this is the first meta-analysis evaluating the value of CTCs in these patients. Overall, our results demonstrated that patients in CTC-positive group had a worse OS and PFS/DFS compared with CTC- negative group; moreover, the presence of CTCs was associated with elevated CA125. 
Study

ID
$\%$

Weight

Fan (2009)

Poveda (2011)

Obermayr (2013)

Sang (2014)

Pearl (2014)

Overall (l-squared $=0.0 \%, p=0.674$ )

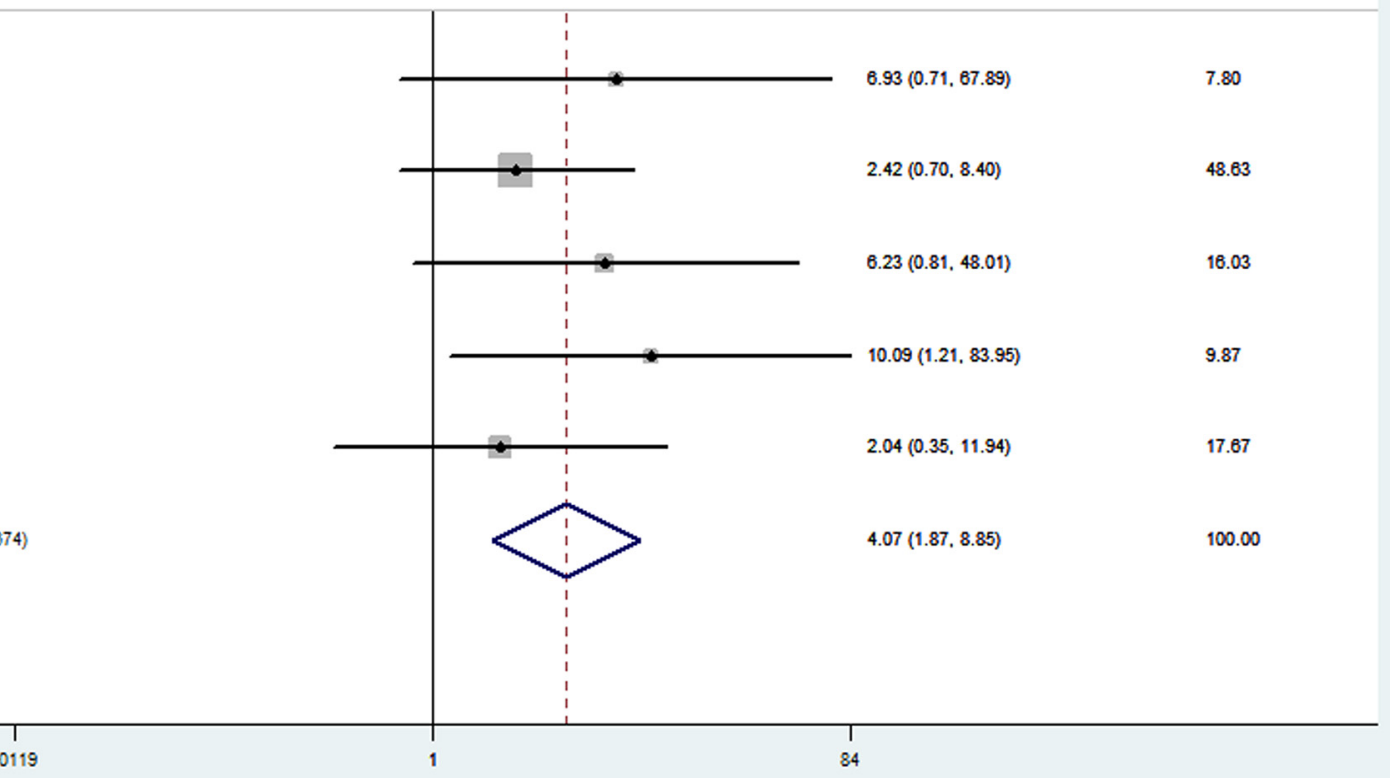

Fig 6. Forest plots of the association between CTCs and CA-125. CTCs = circulating tumor cells.

doi:10.1371/journal.pone. $0130873 . g 006$

Subgroup analysis showed that "RT-PCR" subgroup presented significant association between CTCs and OS, whereas it was not significant in the "CellSearch" subgroup and "Other ICC" subgroup. But the detection method didn't influence HR estimates in the meta-regression $(\mathrm{P}=0.118)$. Though the CellSearch system which uses EpCAM for cell isolation is the only CTC test approved by US FDA for clinical use currently, it has some limitations. EpCAM can be downregulated by cancer stem cells in the procedure of epithelial mesenchymal transition [24]. Thus this system suffers from relatively low sensitivity, additional innovative detection methods may reveal more tumor cells [25]. On the other hand, CellSearch system may also present poor specificity because of biologic nonspecificity [26]. Therefore the methodologies which do not use epithelial markers alone for cell isolation may be more likely to associate with OS according to our subgroup analysis.

In addition, subgroup analysis based on treatment methods showed the prognostic value of CTCs for OS was significant in the "Surgery" subgroup, while it was not significant in the "Chemotherapy" subgroup. However, it is difficult to determine whether treatment methods influence the prognostic value of CTCs. The reason for the drawback is that there was a notable difference in timing for CTCs detection, response to treatment, chemotherapy regimens and operative plans. Further studies are required to investigate the prognostic relevant factor. 


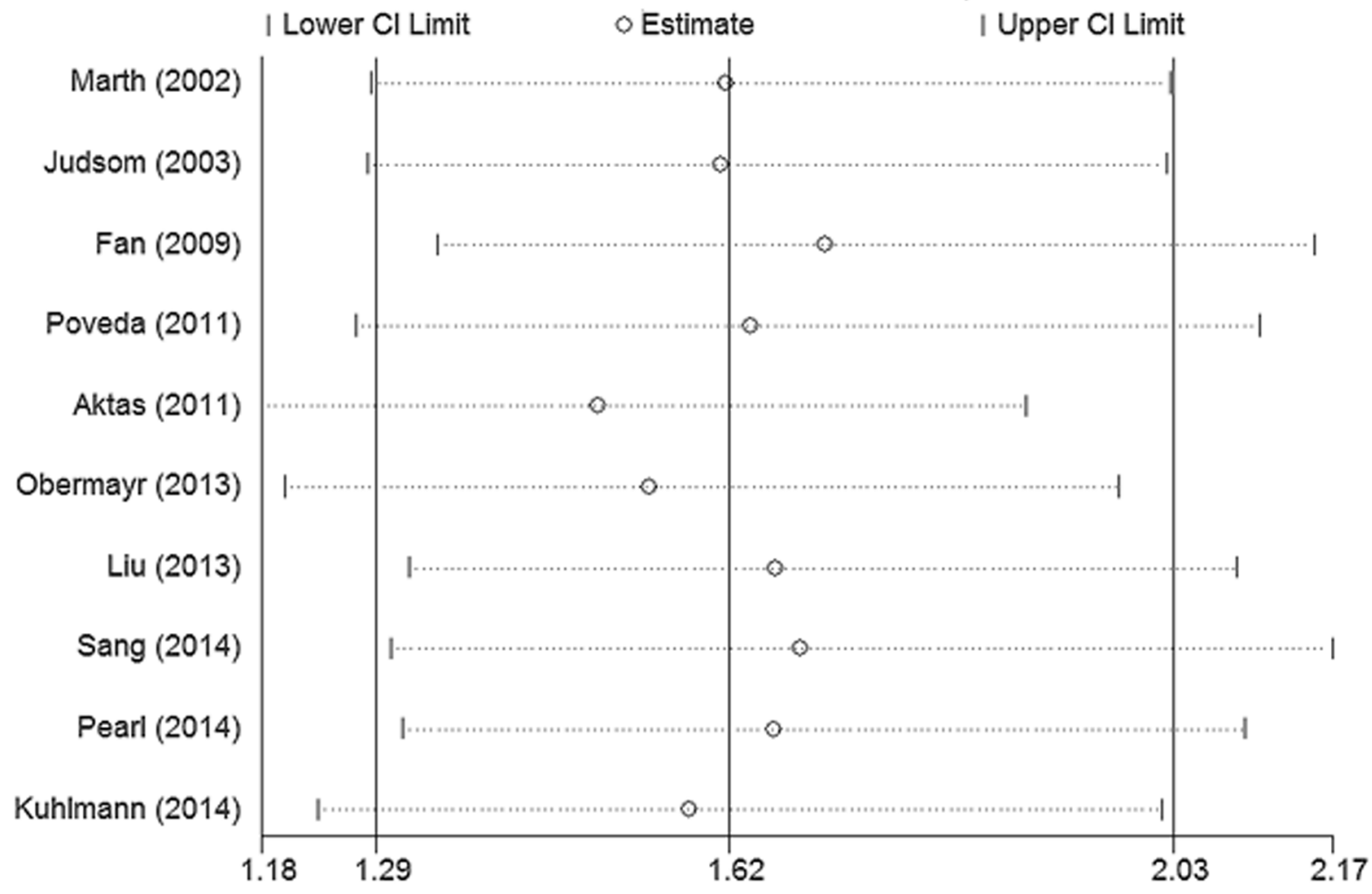

Fig 7. Sensitivity analysis for the pooled HRs in OS. The analysis was conducted by estimating the average HR in the absence of each study. HRs = hazard ratios; OS = overall survival.

doi:10.1371/journal.pone.0130873.g007

To explore potential causes of heterogeneity, we performed meta-regression to evaluate whether percentage of advanced stage patients (stage IV or stage III) would influence the prognostic value of CTCs. No association could be observed ( $\mathrm{p}=0.645)$, which meant the prognostic value of CTCs was not associated with disease stage. Moreover, no significant association could be observed in publication year (0.619) treatment type (0.469) and sample size (0.059).

Our meta-analysis also confirmed the presence of CTC was closely associated with elevated CA-125, both of which are known to be prognostic tool in ovarian cancer.

Our study has some limitations. First, CTC detection methods were different among included studies, which may partly influence the significance in survival analyses. Second, there is no consensus on the optimal cutoff of CTCs for predicting the clinical outcome in ovarian cancer. The low cutoff of $\geq 2 \mathrm{CTC} / 7.5 \mathrm{ml}$ of blood with the CellSearch system was used in three studies [14,16,19], and other studies used different cutoffs. Though Fan et al. showed higher CTC counts could reflect later stage disease and higher CA-125 levels [20], few trials had evaluated by the prognostic value of different numbers of enumerating CTCs in patients with ovarian cancer. Further studies are required to assess prognostic relevant CTC cutoff levels. Third, some studies included in the meta-analysis were small case-series, thus more large prospective studies should be performed.

In conclusion, this meta-analysis indicates that CTC status is associated with OS and PFS/ DFS in patients with ovarian cancer. To achieve clinical utility of CTCs in ovarian cancer, more large prospective studies should be conducted before CTC detection can be applied to clinical use as a prognostic indication. 
Begg's funnel plot with pseudo $95 \%$ confidence limits

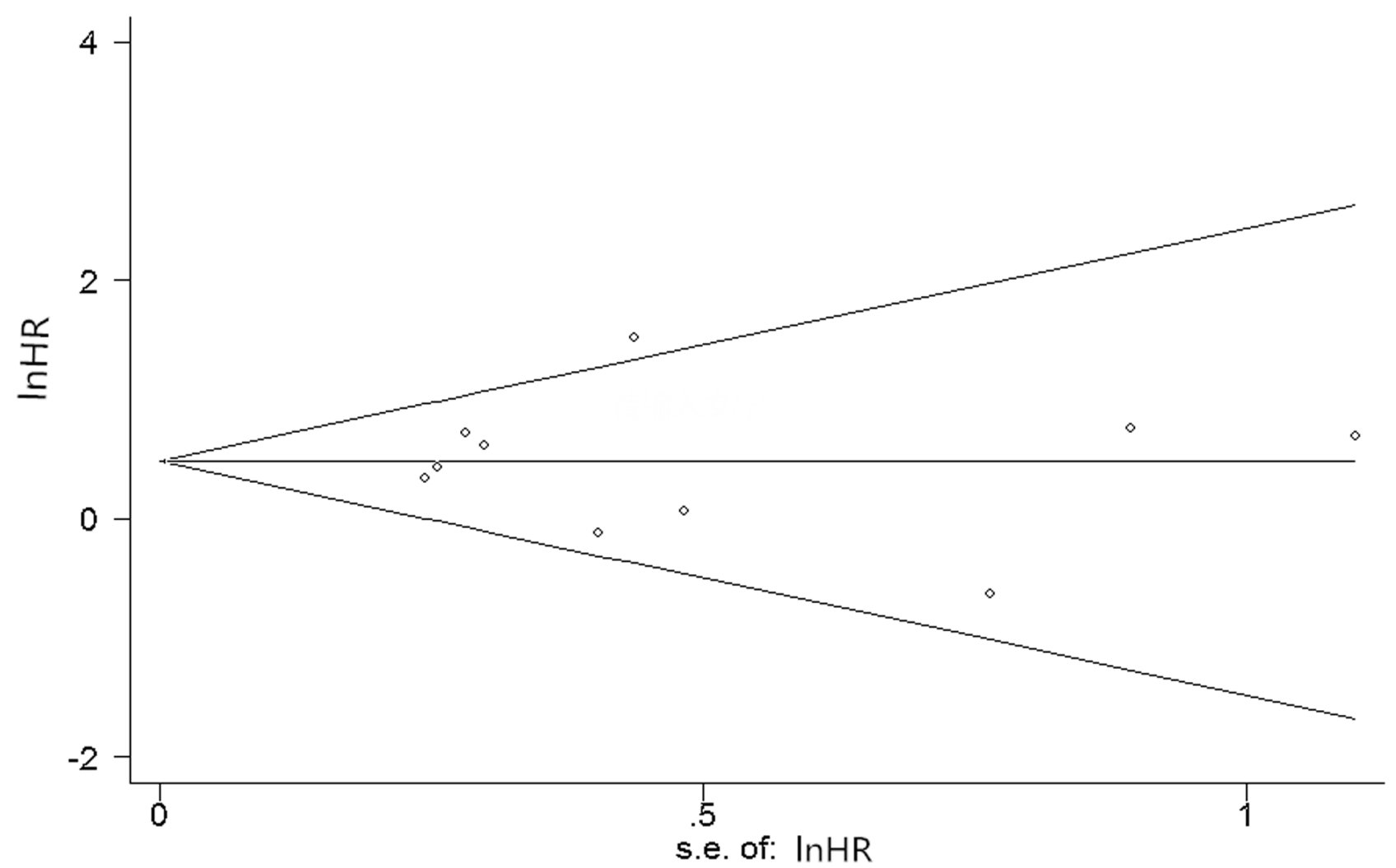

Fig 8. Begg's funnel plots of the prognostic role of CTCs in OS. CTCs = circulating tumor cells; OS = overall survival.

doi:10.1371/journal.pone.0130873.g008

\section{Supporting Information}

S1 Checklist. PRISMA checklist.

(DOC)

S2 Checklist. Meta-analysis on genetic association studies checklist.

(DOC)

S1 Table. The excluded articles and the reasons for exclusion.

(DOC)

S2 Table. Results of meta-regression analysis exploring source of heterogeneity with overall survival.

(DOC)

\section{Author Contributions}

Conceived and designed the experiments: LS. Performed the experiments: YZ BB. Analyzed the data: YZ BB XY GX YM. Contributed reagents/materials/analysis tools: YZ BB XY GX YM.

Wrote the paper: $\mathrm{YZ} B \mathrm{BB}$. 


\section{References}

1. International Agency for Research on Cancer. GLOBOCAN 2012: Estimated Cancer Incidence, Mortality and Prevalence Worldwide in 2012. Database: GLOBOCAN [Internet]. Available: http://globocan. iarc.fr.

2. Jacobs IJ, Menon U. Progress and challenges in screening for early detection of ovarian cancer. Mol Cell Proteomics. 2004; 3(4): 355-366. PMID: 14764655

3. Sevinc A, Adli M, Kalender ME, Camci C. Benign causes of increased serum CA-125 concentration. Lancet Oncol. 2007; 8(12): 1054-1055. PMID: 18054877

4. Zhang L, Riethdorf S, Wu G, Wang T, Yang K, Peng G, et al. Meta-Analysis of the prognostic value of circulating tumor cells in breast cancer. Clinical Cancer Research. 2012; 18(20): 5701-5710. doi: 10. 1158/1078-0432.CCR-12-1587 PMID: 22908097

5. Groot Koerkamp B, Rahbari NN, Büchler MW, Koch M, Weitz J. Circulating tumor cells and prognosis of patients with resectable colorectal liver metastases or widespread metastatic colorectal cancer: a meta-analysis. Ann Surg Oncol. 2013; 20(7): 2156-2165. doi: 10.1245/s10434-013-2907-8 PMID: 23456317

6. Wang S, Zheng G, Cheng B, Chen F, Wang Z, Chen Y, et al. Circulating tumor cells (CTCs) detected by RT-PCR and its prognostic role in gastric cancer: a meta-analysis of published literature. PLoS One. 2014; 9(6): e99259. doi: 10.1371/journal.pone.0099259 PMID: 24901848

7. Wang J, Wang $\mathrm{K}, \mathrm{Xu} \mathrm{J}$, Huang J, Zhang T. Prognostic significance of circulating tumor cells in nonsmall-cell lung cancer patients: a meta-analysis. PLoS One. 2013; 8(11): e78070. doi: 10.1371/journal. pone.0078070 PMID: 24223761

8. Han $L$, Chen W, Zhao Q. Prognostic value of circulating tumor cells in patients with pancreatic cancer: a meta-analysis. Tumour Biol. 2014; 35(3): 2473-2480. doi: 10.1007/s13277-013-1327-5 PMID: 24218336

9. Tierney JF, Stewart LA, Ghersi D, Burdett S, Sydes MR. Practical methods for incorporating summary time-to-event data into meta-analysis. Trials. 2007; 8: 16. PMID: 17555582

10. Whitehead A. Meta-analysis of controlled clinical trial. 1st ed. Chichester: John Wiley \& Sons, Ltd; 2002.

11. Borenstein M, Hedges LV, Higgins JPT, Rothstein HR. Introduction to meta-Analysis. 1st ed. Chichester: John Wiley \& Sons, Ltd; 2009.

12. McGauran N, Wieseler $B$, Kreis J, Schüler YB, Kölsch $H$, Kaiser $T$, et al. Reporting bias in medical research—a narrative review. Trials. 2010; 11: 37. doi: 10.1186/1745-6215-11-37 PMID: 20388211

13. Marth C, Kisic J, Kaern J, Tropé C, Fodstad $\varnothing$. Circulating tumor cells in the peripheral blood and bone marrow of patients with ovarian carcinoma do not predict prognosis. Cancer. 2002; 94(3): 707-712. PMID: 11857303

14. Behbakht K, Sill MW, Darcy KM, Rubin SC, Mannel RS, Waggoner S, et al. Phase II trial of the mTOR inhibitor, temsirolimus and evaluation of circulating tumor cells and tumor biomarkers in persistent and recurrent epithelial ovarian and primary peritoneal malignancies: a Gynecologic Oncology Group study. Gynecol Oncol. 2011; 123(1): 19-26. doi: 10.1016/j.ygyno.2011.06.022 PMID: 21752435

15. Judson PL, Geller MA, Bliss RL, Boente MP, Downs LS Jr, Argenta PA, et al. Preoperative detection of peripherally circulating cancer cells and its prognostic significance in ovarian cancer. Gynecol Oncol. 2003, 91(2): 389-394. PMID: 14599871

16. Liu JF, Kindelberger David, Doyle Courtney, Lowe A, Barry WT, Matulonis UA. Predictive value of circulating tumor cells (CTCs) in newly-diagnosed and recurrent ovarian cancer patients. Gynecol Oncol. 2013, 131(2): 352-356. doi: 10.1016/j.ygyno.2013.08.006 PMID: 23954902

17. Sang M, Wu X, Fan X, Sang M, Zhou X, Zhou N. Multiple MAGE-A genes as surveillance marker for the detection of circulating tumor cells in patients with ovarian cancer. Biomarkers. 2014; 19(1): 34-42. doi: 10.3109/1354750X.2013.865275 PMID: 24320162

18. Pearl ML, Zhao Q, Yang J, Dong H, Tulley S, Zhang Q, et al. Prognostic analysis of invasive circulating tumor cells (iCTCs) in epithelial ovarian cancer. Gynecol Oncol. 2014; 134(3): 581-590. doi: 10.1016/j. ygyno.2014.06.013 PMID: 24972191

19. Poveda A, Kaye SB, McCormack R, Wang S, Parekh T, Ricci D, et al. Circulating tumor cells predict progression free survival and overall survival in patients with relapsed/recurrent advanced ovarian cancer. Gynecol Oncol. 2011; 122(3): 567-572. doi: 10.1016/j.ygyno.2011.05.028 PMID: 21664658

20. Fan T, Zhao Q, Chen JJ, Chen WT, Pearl ML. Clinical significance of circulating tumor cells detected by an invasion assay in peripheral blood of patients with ovarian cancer. Gynecol Oncol. 2009; 112(1): 185-191. doi: 10.1016/j.ygyno.2008.09.021 PMID: 18954898 
21. Aktas B, Kasimir-Bauer S, Heubner M, Kimmig R, Wimberger P. (2011) Molecular profiling and prognostic relevance of circulating tumor cells in the blood of ovarian cancer patients at primary diagnosis and after platinum-based chemotherapy. Int J Gynecol Cancer 21(5): 822-830. doi: 10.1097/IGC. Ob013e318216cb91 PMID: 21613958

22. Obermayr E, Castillo-Tong DC, Pils D, Speiser P, Braicu I, Van Gorp T, et al. Molecular characterization of circulating tumor cells in patients with ovarian cancer improves their prognostic significance-a study of the OVCAD consortium. Gynecol Oncol. 2013; 128(1): 15-21. doi: 10.1016/j.ygyno.2012.09.021 PMID: 23017820

23. Kuhlmann JD, Wimberger P, Bankfalvi A, Keller T, Schöler S, Aktas B, et al. ERCC1-positive circulating tumor cells in the blood of ovarian cancer patients as a predictive biomarker for platinum resistance. Clin Chem. 2014; 60(10): 1282-1289. doi: 10.1373/clinchem.2014.224808 PMID: 25015375

24. Mani SA, Guo W, Liao MJ, Eaton EN, Ayyanan A, Zhou AY, et al. The epithelial-mesenchymal transition generates cells with properties of stem cells. Cell. 2008; 133(4): 704-715. doi: 10.1016/j.cell.2008. 03.027 PMID: 18485877

25. Yu M, Stott S, Toner M, Maheswaran S, Haber DA. Circulating tumor cells:approaches to isolation and characterization. J Cell Biol. 2011; 192(3): 373-382. doi: 10.1083/jcb.201010021 PMID: 21300848

26. Wicha MS, Hayes DF. Circulating tumor cells: not all detected cells are bad and not all bad cells are detected. J Clin Oncol. 2011; 29(12): 1508-1511. doi: 10.1200/JCO.2010.34.0026 PMID: 21422428 